\title{
Avaliação da qualidade de vida e relação com o nível de atividade física em idosos utilizando os questionários WHOQOL-bref e IPAQ.
}

\author{
Physical activity and quality of life evaluation in the elderly people using \\ WHOQOI-bref ad IPAQ questionnaires
}

\author{
1 Eduardo Villar Guimarães eduardovillarg@gmail.com \\ 1 Henrique Pereira da Rosa Silva \\ 2 Rubens Basile
}

\footnotetext{
Discente do Curso de Medicina do UniFOA

Mestre em Medicina pela UFRJ, docente do Curso de Medicina do UniFOA
}

\section{Resumo}

Nas últimas décadas, o Brasil apresentou um aumento significativo de idosos e as projeções estatísticas apontam que, em 2025, cerca de $15 \%$ de sua população será constituída por esse grupo. Este trabalho objetivou avaliar a qualidade de vida de idosos praticantes de atividade física e residentes no município de Volta Redonda, no estado do Rio de Janeiro, justificando-se pelo rápido aumento da população idosa sem a correspondente modificação desse aspecto, considerando também o impacto que a atividade física regular pode ter na melhora desse indicador. Foram selecionados 20 pacientes atendidos nas Unidades Básicas de Saúde da Família dos bairros Ponte Alta, São Carlos e Três Poços. Para avaliação da qualidade de vida, optou-se por dois questionários autoaplicáveis, previamente validados para a população brasileira e por um questionário sociodemográfico. Apesar do estudo contar com uma amostra pequena, não houve diferença significativa na qualidade de vida em geral entre a população estudada e os estudos descritos.

\section{Palavras-chave}

Qualidade de vida. Exercício. Idoso.

\begin{abstract}
Aging is an intrinsic, active and progressive process, followed by physical, physiological and psychological changes. In the last decades, Brazil has shown a significant increase in the elderly and the statistics projections point out that in 2025 nearly 15\% of its population will be composed by this group. The aim of this work was to evaluate the quality of life and the physical activity relations in the elderly people living in the city of Volta Redonda, in the state of Rio de Janeiro, Brazil. It was justified by the rapid increasing in the elderly population without the correspondent improving in the quality of life and the impact that regular physical activity may have in this measurement. This study was performed with 20 patients in three Family Healthcare Units in different regions, using the World Health Organization Quality of Life form and the International Physical Activity Questionary (IPAQ). There was no significant difference in quality of life between this group and other similar studies.
\end{abstract}

\section{Keywords}

Quality of life. Exercise. Aged.

\section{Como você deve citar?}

GUIMARÃES, Eduardo Villar; SILVA, Henrique Pereira da Rosa; BASILE, Rubens. Avaliação da qualidade de vida e relação com o nível de atividade física em idosos utilizando os questionários WHOQOL-bref e IPAQ. Cadernos UniFOA, Volta Redonda, n. 43, p. 133-141, agosto. 2020. 


\section{INTRODUÇÃO}

O envelhecimento se constitui como um processo intrínseco, ativo e progressivo, acompanhado por alterações físicas, fisiológicas e psicológicas, as quais podem acarretar prejuízos na capacidade do idoso em se adaptar ao meio em que vive (MOURÃO; SILVA, 2010).

$\mathrm{Na}$ atualidade, o envelhecimento populacional é um fenômeno mundial. Não diferentemente, o Brasil apresenta um aumento significativo de idosos, demonstrado nos dados demográficos: em 2000, os idosos representavam 8,6\% do total da população; já em 2010, eles constituíam 10,7\%. As projeções estatísticas apontam que, em 2025, o Brasil deverá ter $15 \%$ de sua população constituída por idosos, o que colocará o país em sexto lugar no ranking mundial (VICENTE; SANTOS, 2013).

Nesse processo, a adoção de hábitos de vida saudável, como a prática de atividade física, promove a saúde ao longo da vida, influenciando o envelhecimento de maneira positiva e a melhor percepção da qualidade de vida, que contempla aspectos multifatoriais das facetas físicas, psicológicas, sociais e ambientais (SONATI et al., 2014).

Essa prática regular de atividades físicas durante o envelhecimento pode melhorar a autoestima, a funcionalidade e os aspectos psicológicos e sociais dos idosos, diminuindo a ansiedade e a tensão. Ademais, há evidências de que idosos fisicamente ativos apresentam uso reduzido de medicamentos, além de apresentarem menores sintomas depressivos e estresse (GUIMARÃES et al., 2012).

Além disso, a prática de atividades físicas também está consistentemente associada aos seguintes domínios de qualidade de vida: capacidade funcional, qualidade de vida em geral, autonomia, atividades passadas, presentes e futuras, intimidade, saúde mental, vitalidade e psicológico. Essas associações podem estar relacionadas ao fato de que a atividade física promove independência, melhorando a capacidade funcional e saúde física, essenciais para a autonomia individual. (VAGETTI et al., 2014).

Mediante essas informações, nota-se que a atividade física tem um papel importante nesse processo de envelhecimento e que a sua prática regular impacta diretamente na qualidade de vida. 0 processo de envelhecimento sedentário pode gerar uma série de comprometimentos físicos e psíquicos, os quais acarretam a redução na qualidade de vida. Em contraponto, o envelhecimento ativo associado à prática regular de atividades físicas pode gerar uma melhora nos indicadores de saúde e, da mesma forma, na qualidade de vida.

Desse modo, mostrou-se relevante avaliar e mensurar a percepção e o impacto dessas atividades na qualidade de vida de idosos praticantes de atividade física residentes no município de Volta Redonda, no estado do Rio de Janeiro, utilizando-se a versão brasileira do questionário da Organização Mundial da Saúde WHOQOL - bref e correlacionar os dados com os obtidos por meio do Questionário Internacional de Atividade Física - Versão Curta (International Physical Activity Questionnaire - IPAQ).

\section{MATERIAIS E MÉTODOS}

O estudo teve características do tipo descritivo transversal e foi realizado nas Unidades Básicas de Saúde da Família (UBSF) dos bairros Ponte Alta, São Carlos e Três Poços, em Volta Redonda, interior do estado do Rio de Janeiro. 
Foram selecionados 20 pacientes, através de uma amostragem não probabilística consecutiva de todos que preencheram os critérios de inclusão do estudo no período de setembro a dezembro de 2018, até que o tamanho da amostra foi alcançado.

Foram incluídos pacientes maiores de 60 anos, fisicamente independentes, de ambos os sexos, residentes no município de Volta Redonda, no estado do Rio de Janeiro, e foram excluídos pacientes que não aceitaram participar do estudo e não assinaram o Termo de Consentimento Livre e Esclarecido ou que foram incapazes de responder adequadamente ao questionário.

Para a avaliação da qualidade de vida, foi utilizado nesta pesquisa a versão brasileira do instrumento World Health Organization Quality of Life Assessment (Whoqol - bref), questionário multidimensional desenvolvido pela Organização Mundial da Saúde (OMS), composto de 26 questões, cujas respostas geram pontuações que variam de 1 a 5 , sendo duas questões sobre a qualidade de vida geral; sete questões que compõem o domínio físico; seis, o domínio psicológico; três, o domínio relações sociais; e oito, o domínio meio ambiente. Os domínios e facetas do questionário são descritos na Tabela 1.

Tabela 1 - Domínios e facetas do WHOQOL-bref

\begin{tabular}{l}
\hline \multicolumn{1}{c}{ Domínio I - Domínio físico } \\
\hline Dor e desconforto \\
\hline Energia e fadiga \\
\hline Sono e repouso \\
\hline Mobilidade \\
\hline Atividades da vida cotidiana \\
\hline Dependência de medicação ou de tratamentos \\
\hline Capacidade de trabalho \\
\hline Domínio II - Domínio psicológico \\
\hline Sentimentos positivos \\
\hline Pensar, aprender, memória e concentração \\
\hline Autoestima \\
\hline Imagem corporal e aparência \\
\hline Sentimentos negativos \\
\hline Espiritualidade/religião/crenças pessoais \\
\hline Domínio III - Relações sociais \\
\hline Relações pessoais \\
\hline Suporte (Apoio) social \\
\hline Atividade sexual \\
\hline Domínio IV - Meio ambiente \\
\hline Segurança física e proteção \\
\hline Ambiente no lar \\
\hline Recursos financeiros \\
\hline Cuidados de saúde e sociais: disponibilidade e qualidade \\
\hline Oportunidades de adquirir novas informações e habilidades \\
\hline Participação em, e oportunidades de recreação/lazer \\
\hline Ambiente físico: (poluição/ruído/trânsito/clima) \\
\hline Transporte \\
\hline
\end{tabular}

Fonte: FLECK (2000) 
Juntamente com o instrumento semiestruturado, foi administrado o Questionário Internacional de Atividade Física - Versão Curta (IPAQ), também desenvolvido pela Organização Mundial da Saúde, cujos resultados, de acordo com a orientação do próprio IPAQ, podem ser classificados em:

Sedentário: indivíduo que não realiza nenhuma atividade física por, pelo menos, 10 minutos contínuos durante a semana;

Insuficientemente Ativo: consiste em classificar os indivíduos que praticam atividades físicas por, pelo menos, 10 minutos contínuos por semana, porém de maneira insuficiente para serem classificados como ativos. Para classificar os indivíduos nesse critério, são somadas a duração e a frequência dos diferentes tipos de atividades (caminhadas + moderada + vigorosa).

Ativo: cumpre as seguintes recomendações: a) atividade física vigorosa $\geq 3$ dias/semana e $\geq$ 20 minutos/sessão; b) moderada ou caminhada $\geq 5$ dias/semana e $\geq 30$ minutos/sessão; c) qualquer atividade somada: $\geq 5$ dias/semana e $\geq 150 \mathrm{~min} / \mathrm{semana}$;

Muito Ativo: cumpre as seguintes recomendações: a) vigorosa $\geq 5$ dias/semana e $\geq 30 \mathrm{~min} / \mathrm{ses}$ são; b) vigorosa $\geq 3$ dias/ semana e $\geq 20 \mathrm{~min} /$ sessão + moderada e ou caminhada $\geq 5$ dias/ semana e $\geq 30 \mathrm{~min} /$ sessão.

Além desses dois questionários, os dados sociodemográficos da amostra foram colhidos por meio de um formulário, no qual foram analisados sexo, idade, estado civil e nível de escolaridade.

Os dados quantitativos de todos os questionários foram avaliados através do programa Microsoft Office Excel $2016{ }^{\circledR}$, utilizando-se estatística descritiva por meio de tabela de frequência, média aritmética e seus respectivos desvios-padrões. 0 trabalho foi aprovado pelo Comitê de Ética em Pesquisa em Seres Humanos do UniFOA, sob o CAAE 91687118.4.0000.5237.

\section{RESULTADOS}

Este estudo teve como amostra um grupo de 20 indivíduos selecionados, de acordo com os critérios de inclusão. Desse grupo, nenhum entrevistado foi excluído da amostra. As demais características sociodemográficas dos entrevistados, que incluem idade, sexo, escolaridade e estado civil, foram descritas na Tabela 2. 
Tabela 2 - Características sociodemográficas dos entrevistados

\begin{tabular}{ll}
\hline Características & Resultados \\
\hline Idade (anos) & $68,25 \pm 7,46$ \\
\hline Sexo Feminino (\%) & 80 \\
\hline Sexo Masculino (\%) & 20 \\
\hline Estado Civil: & 15,79 \\
\hline Solteiro (\%) & 0 \\
\hline Separado (\%) & 31,58 \\
\hline Casado (\%) & 26,32 \\
\hline Viúvo (\%) & 26,31 \\
\hline Divorciado (\%) & \\
\hline Escolaridade: & 30 \\
\hline Nenhuma (\%) & 45 \\
\hline Primário completo (\%) & 15 \\
\hline Ginásio completo (\%) & 5 \\
\hline Ensino Médio completo (\%) & 5 \\
\hline Superior completo (\%) & 18,52 \\
\hline Vive: & 29,63 \\
\hline Sozinho (\%) & 33,33 \\
\hline Cônjuge (\%) & 18,52 \\
\hline Filhos (\%) & \\
\hline Netos (\%) & \\
\hline
\end{tabular}

Fonte: dos autores, 2019.

Para análise da qualidade de vida por meio do WHOQOL-BREF, inicialmente, foi calculado um Escore Bruto (EB) mediante a soma das pontuações de cada questão e posteriormente foi gerado um Escore Transformado 4-20 (ET 4-20), cujos valores variam de 4 a 20, conforme mostrado na Tabela 3.

Tabela 3 - Resultados obtidos para cada domínio do questionário WHOQOL - bref

\begin{tabular}{lcccccc}
\hline DOMÍNIO & MÉDIA & $\begin{array}{c}\text { DESVIO } \\
\text { PADRÃO }\end{array}$ & $\begin{array}{c}\text { COEFICIENTE DE } \\
\text { VARIAÇÃO }\end{array}$ & $\begin{array}{c}\text { VALOR } \\
\text { MÍNIMO }\end{array}$ & $\begin{array}{c}\text { VALOR } \\
\text { MÁXIMO }\end{array}$ & AMPLITUDE \\
\hline Físico & 14,57 & 2,61 & 17,93 & 9,71 & 17,71 & 8,00 \\
\hline Psicológico & 15,60 & 2,68 & 17,17 & 10,00 & 19,33 & 9,33 \\
\hline Relações Sociais & 14,27 & 3,09 & 21,68 & 6,67 & 20,00 & 13,33 \\
\hline Meio Ambiente & 13,80 & 2,57 & 18,64 & 9,50 & 18,50 & 9,00 \\
\hline Autoavaliação da QV & 13,30 & 2,36 & 17,78 & 8,00 & 16,00 & 8,00 \\
\hline TOTAL & 14,44 & 1,89 & 13,06 & 10,15 & 17,85 & 7,69 \\
\hline
\end{tabular}

Fonte: dos autores, 2019.

Na sequência, calculou-se o Escore Transformado 0-100 (ET 0-100), no qual os valores variam de 0 a 100. Esses resultados estão descritos no Gráfico 1. 
Gráfico 1 - Escore dos domínios do questionário WHOQOL - bref

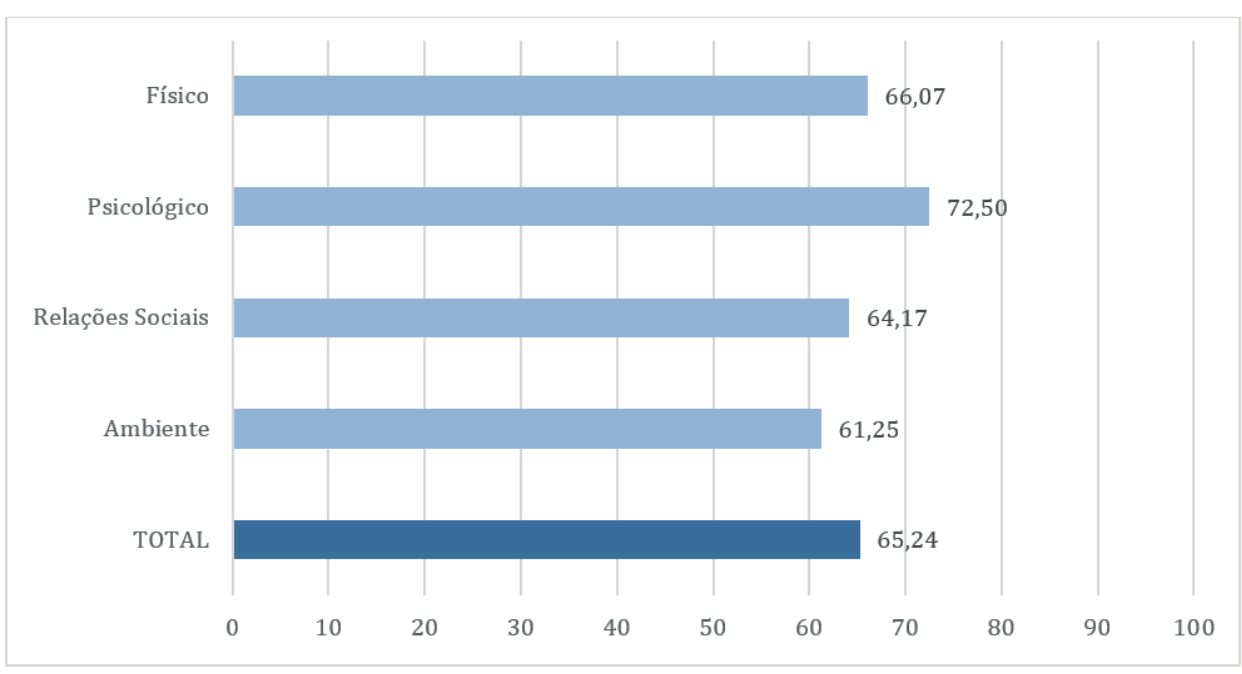

Fonte: dos autores, 2019.

Os dados obtidos e classificação de acordo com as respostas dadas ao questionário internacional de atividade física (IPAQ) estão descritos na Tabela 4.

Tabela 4 - Resultados obtidos pelo questionário IPAQ

\begin{tabular}{llllllll}
\hline \multirow{2}{*}{ Indivíduos } & \multicolumn{2}{l}{ Caminhada } & \multicolumn{2}{l}{ Moderada } & \multicolumn{2}{l}{ Vigorosa } & Classificação \\
\cline { 2 - 7 } & $\mathbf{F}$ & $\mathbf{D}$ & $\mathbf{F}$ & $\mathbf{D}$ & $\mathbf{F}$ & $\mathbf{D}$ & \\
\hline 1 & 3 & 30 & 0 & 0 & 0 & 0 & Insuficientemente Ativo \\
\hline 2 & 5 & 180 & 3 & 60 & 0 & 0 & Ativo \\
\hline 4 & 7 & 30 & 0 & 0 & 0 & 0 & Ativo \\
\hline 5 & 7 & 60 & 0 & 0 & 0 & 0 & Ativo \\
\hline 6 & 7 & 30 & 3 & 90 & 0 & 0 & Ativo \\
\hline 7 & 3 & 15 & 7 & 30 & 0 & 0 & Ativo \\
\hline 8 & 5 & 60 & 7 & 60 & 0 & 0 & Ativo \\
\hline 9 & 3 & 30 & 3 & 180 & 0 & 0 & Ativo \\
\hline 10 & 3 & 30 & 2 & 90 & 0 & 0 & Ativo \\
\hline 11 & 4 & 60 & 0 & 0 & 0 & 0 & Insuficientemente Ativo \\
\hline 12 & 7 & 60 & 1 & 20 & 0 & 0 & Ativo \\
\hline 13 & 7 & 10 & 2 & 60 & 0 & 0 & Ativo \\
\hline 14 & 2 & 20 & 7 & 10 & 0 & 0 & Insuficientemente Ativo \\
\hline 15 & 6 & 30 & 3 & 60 & 0 & 0 & Ativo \\
\hline 16 & 1 & 30 & 0 & 0 & 0 & 0 & Insuficientemente Ativo \\
\hline 17 & 7 & 120 & 7 & 180 & 0 & 0 & Ativo \\
\hline 18 & 2 & 30 & 7 & 20 & 0 & 0 & Ativo \\
\hline 19 & 7 & 180 & 7 & 180 & 0 & 0 & Ativo \\
\hline 20 & 30 & 7 & 20 & 0 & 0 & Ativo \\
\hline & 3 & 30 & 0 & 0 & 0 & 0 & Ativo \\
\hline
\end{tabular}

Nota: $F$ - frequência (dias), D - duração (minutos/dia) 
Tabela 5 - Resultados obtidos pelo questionário IPAQ por classificação

\begin{tabular}{ll}
\hline Classificação & Resultados (\%) \\
\hline Muito Ativo & 0 \\
\hline Ativo & 80 \\
\hline Insuficientemente Ativo & 20 \\
\hline Sedentário & 0 \\
\hline
\end{tabular}

Fonte: dos autores, 2019.

\section{DISCUSSÃO}

Neste estudo, avaliou-se a qualidade de vida dos usuários atendidos em três unidades básicas de saúde família (UBSF) do município de Volta Redonda. As características sociodemográficas da população entrevistada nessas unidades são semelhantes as encontradas por Tavares et al. (2016) e por Almeida-Brasil et al. (2017). Os resultados também estão de acordo com o estudo realizado por Guibu et al. (2017) com 8.676 usuários em serviços de atenção primária de amostra representativa de municípios, estratificada pelas regiões do Brasil, no qual o perfil predominante dos usuários da atenção primária, entre outras variáveis, é, em grande maioria, de mulheres com baixa escolaridade.

Para a análise dos resultados do questionário WHOQOL-bref, a classificação descrita por Gomes et al. (2014), no sentido de facilitar os cálculos, considerou como ponto de corte o valor abaixo de 70 e igual ou maior que 70 , em que níveis abaixo de 70 são considerados como insatisfação, e níveis acima, como satisfação com wa qualidade de vida. Entretanto, o estudo realizado por Silva et al. (2019) mostrou que o ponto de corte de menor que 60 apresentou alta sensibilidade e excelente valor preditivo negativo para baixa qualidade de vida. No presente estudo, foi utilizado o valor menor que 60 como ponto de corte para baixa qualidade de vida.

As médias obtidas da qualidade de vida nos domínios Físico, Relações Sociais e Qualidade de Vida geral foram semelhantes as encontradas por Almeida-Brasil et al. (2017), em estudo realizado em quatro Unidades Básicas de Saúde (UBS) de Belo Horizonte, Minas Gerais, com 930 usuários adultos maiores de 18 anos. Os domínios Psicológico e Ambiente apresentaram resultados superiores no presente trabalho.

Outro estudo realizado por Silva et al. (2019), com o objetivo de determinar pontos de corte para detectar uma baixa qualidade de vida pelo questionário WHOQOL-bref em indivíduos idosos, na região administrativa de Samambaia, Distrito Federal, com 466 indivíduos, apresentou os resultados mostrados na Tabela 6.

Tabela 6 - Resultados obtidos por Silva et al. (2019) e pelo presente estudo

\begin{tabular}{lll}
\hline Domínios & Silva et al. (2019) & Dados da pesquisa \\
\hline Físico & 62,9 & 66,07 \\
\hline Psicológico & 65,2 & 72,5 \\
\hline Relações Sociais & 63,3 & 64,17 \\
\hline Ambiente & 53,3 & 61,25 \\
\hline Total & 61,2 & 65,24 \\
\hline
\end{tabular}

Fonte: Silva et al. (2019); dos autores, 2019. 
Em comparação com o estudo supracitado, o presente estudo, novamente, apresentou resultados superiores nos domínios Psicológico e Ambiente aos encontrados em Samambaia, Distrito Federal.

Em relação aos resultados encontrados pela aplicação do IPAQ, Macedo et al. (2015) verificou em estudo realizado em Curitiba, Paraná, com 173 participantes com média de idade de 69,9 anos, que $81,11 \%$ da amostra total são sedentários ou irregularmente ativos, resultado que difere do presente estudo, em que $80 \%$ dos indivíduos são considerados ativos, seguindo os mesmos critérios.

Dos indivíduos entrevistados, não houve significativa diferença dos valores da qualidade de vida total entre os indivíduos classificados como ativos ou insuficientemente ativos, entretanto os ativos apresentaram maior resultado nos domínios físico e relações sociais, o que evidencia os benefícios já conhecidos da prática de atividades físicas. Ambos os grupos (ativos e insuficientemente ativos) apresentaram como menores resultados o domínio Ambiente. A relação entre os resultados dos dois questionários está descrita na Tabela 7.

Tabela 7 - Relação dos domínios dos WHOQOL-bref e a da classificação do IPAQ

\begin{tabular}{lll}
\hline & Ativo & Insuficientemente Ativo \\
\hline Físico & 66,74 & 63,39 \\
\hline Psicológico & 71,88 & 75 \\
\hline Relações Sociais & 65,1 & 60,42 \\
\hline Ambiente & 61,33 & 60,94 \\
\hline Total & 65,26 & 65,14 \\
\hline
\end{tabular}

Fonte: dos autores, 2019.

Em ambos os grupos (ativos e insuficientemente ativos), o domínio mais afetado foi o Ambiente. Dessa forma, fica evidente que as políticas que visem à segurança, transporte e, principalmente, inclusão social nos locais estudados ainda não são aplicadas de maneira eficiente, sendo necessárias revisões para que essas pessoas também sejam contempladas

\section{CONCLUSÃO}

Apesar de o estudo contar com uma amostra pequena, não houve diferença na qualidade de vida em geral entre a população estudada e os estudos descritos. 0 nível de atividade física encontrado para a faixa etária foi maior do que de outros estudos encontrados, porém a qualidade de vida total das populações ativas e insuficientemente ativas foi semelhante. 


\section{REFERÊNCIAS}

ALMEIDA-BRASIL, C. C. et al. Qualidade de vida e características associadas: aplicação do WHOQOLBREF no contexto da Atenção Primária à Saúde. Ciênc. saúde coletiva, Rio de Janeiro, v. 22, n. 5, p. 1705-1716, Maio 2017.

FLECK, M. P. A. et al. Aplicação da versão em português do instrumento abreviado de avaliação da qualidade de vida "WHOQOL-bref". Rev. Saúde Pública, São Paulo, v. 34, n. 2, p. 178-183, abril. 2000.

GOMES, J. R. A. A. et al. Aplicação do WHOQOL-BREF em segmento da comunidade como subsídio para ações de promoção da saúde. Rev Bras Epidemiol. v.17, n.2, p.495-516, abril/jun. 2014.

GUIBU, I. A. et al. Características principais dos usuários dos serviços de atenção primária à saúde no Brasil. Rev. Saúde Pública, São Paulo, v. 51, supl. 2, 17s, 2017.

GUIMARÃES, A. C. A. et al. Percepção da qualidade de vida e da finitude de adultos de meia idade e idoso praticantes e não praticantes de atividade física. Revista Brasileira de Geriatria e Gerontologia, Rio de Janeiro, v.15, n.4, p.661-667, out./dez. 2012.

MACEDO, R. M. et al. Nível de atividade física de idosos participantes de um programa de prevenção de doença cardiovascular. ASSOBRAFIR Ciência. v.6, n.3, p.11-20, dez. 2015

MOURÃO, C. A.; SILVA, N. M. Influência de um programa de atividades físicas recreativas na autoestima de idosos institucionalizados. Revista Brasileira de Ciências do Envelhecimento Humano, Passo Fundo, v.7, n.3, p.324-334, set./dez. 2010.

SILVA, S. M. et al. VES-13 and WHOQOL-bref cutoff points to detect quality of life in older adults in primary health care. Rev. Saúde Pública, São Paulo, v. 53, 26, 2019.

SONATI, J. G. et al. Análise comparativa da qualidade de vida de adultos e idosos envolvidos com a prática regular de atividade física. Rev. Bras. Geriatr. Gerontol., Rio de Janeiro, v.17, n.4, p.731-739, out./dez. 2014.

TAVARES, D. M. S. et al . Quality of life and self-esteem among the elderly in the community. Ciênc. saúde coletiva, Rio de Janeiro, v. 21, n. 11, p. 3557-3564, Nov. 2016.

THE WHOQOL GROUP. Development of the World Health Organization WHOQOL-BREF Quality of Life Assessment. Psychological Medicine - Cambridge University Press, Inglaterra, v.28, n.3, p.551-558, 1998.

VAGETTI, G. C. et al. Association between physical activity and quality of life in the elderly: a systematic review. Revista Brasileira de Psiquiatria, São Paulo, v.36, n. 1, p.76-88, jan./mar. 2014.

VICENTE, F. R.; SANTOS, S. M. A. Avaliação multidimensional dos determinantes do envelhecimento ativo em idosos de um município de Santa Catarina. Texto \& Contexto - Enfermagem, Florianópolis, v.22, n.2, p.370-378, abr./jun. 2013.

WORLD HEALTH ORGANIZATION (WHO). Recomendaciones Mundiales Sobre Actividad Física para la Salud. Disponível em: http://whqlibdoc.who.int/publications/2010/9789243599977_spa.pdf. Acesso em 10 maio 2018. 